\title{
A CLASSIFICATION OF SPHERICAL SYMMETRIC CR MANIFOLDS
}

\author{
G. DILEO and A. LOTTA ${ }^{凶}$
}

(Received 16 June 2008)

\begin{abstract}
In this paper we get different characterizations of the spherical strictly pseudoconvex CR manifolds admitting a CR-symmetric Webster metric by means of the Tanaka-Webster connection and of the Riemannian curvature tensor. As a consequence we obtain the classification of the simply connected, spherical symmetric pseudo-Hermitian manifolds.
\end{abstract}

2000 Mathematics subject classification: primary 53C15, 53C25, 53C35; secondary 32V05.

Keywords and phrases: CR-symmetric space, spherical CR manifold, contact Riemannian $(k, \mu)$-space, Bochner curvature tensor.

\section{Introduction}

A spherical CR manifold is a strongly pseudoconvex CR manifold $(M, H M, J)$ of hypersurface type which is locally CR-equivalent to the sphere $\mathbb{S}^{2 n+1}, n=\operatorname{dim}_{C R} M$, endowed with the standard CR structure as a real hypersurface of $\mathbb{C}^{n+1}$. Recall that strong pseudoconvexity means positive definiteness of the Levi form $L_{\eta}$ associated with a suitable global section $\eta$ of the annihilator $H^{o} M$ of the holomorphic tangent bundle of $M$. The 1-form $\eta$ is usually called a pseudo-Hermitian structure on $M$, and it canonically determines a Riemannian metric $g_{\eta}$ which is compatible with the partial complex structure $J: H M \rightarrow H M$ (see, for example, [27, 31]). We shall call $g_{\eta}$ the Webster metric associated with $\eta$. Denoting by $\xi$ the Reeb vector field of the contact form $\eta$, at each point $x \in M$ we have an orthogonal decomposition

$$
T_{x} M=H_{x} M \oplus \mathbb{R} \xi_{x}
$$

with respect to $g_{\eta}$, and moreover $g_{\eta \mid H_{x} M}=\left(L_{\eta}\right)_{x}, g_{\eta}(\xi, \xi)=1$.

Spherical CR manifolds are characterized by $S=0$, where $S$ is the Chern-MoserTanaka pseudoconformal invariant tensor field of type $(1,3)$, and they represent flat spaces among strongly pseudoconvex CR manifolds from the point of view of Cartan geometry (see, for example, [26]). The simply connected, homogeneous

(C) 2009 Australian Mathematical Publishing Association Inc. 0004-9727/2009 \$16.00 
spherical hypersurfaces of the Euclidean space $\mathbb{C}^{n+1}$ were fully classified by Burns and Shnider in [11]. In particular, it is known that the unique compact simply connected homogeneous spherical hypersurface of $\mathbb{C}^{n+1}$ is $\mathbb{S}^{2 n+1}$, up to CR-equivalence. In this paper we adopt a geometric point of view in studying spherical CR manifolds, concentrating our attention on CR-symmetric Webster metrics $g_{\eta}$. For the general notion of a symmetric Hermitian metric on a CR manifold we refer to [19] (see also Section 3). Here we recall that a Webster metric $g_{\eta}$ is CR-symmetric if for each point $x \in M$ there exists a CR-isometry $\sigma: M \rightarrow M$ with $\sigma(x)=x$ and

$$
(d \sigma)_{x \mid H_{x} M}=-\mathrm{Id} .
$$

This notion of course is different from the requirement that $\left(M, g_{\eta}\right)$ be a Riemannian symmetric space, which is known to be quite strong for a Webster metric (see, for example, [15]).

Actually the standard metric $g_{o}$ of curvature 1 on the sphere $\mathbb{S}^{2 n+1}$ is a CRsymmetric Webster metric $g_{\eta_{o}}$ for the choice of a canonical contact form $\eta_{o}$. The symmetry at a point $x \in \mathbb{S}^{2 n+1}$ is the restriction of the unitary reflection $\sigma_{x}(z)=$ $2\langle x, z\rangle x-z$ with respect to the standard Hermitian scalar product of $\mathbb{C}^{n+1}$ (see [19]).

More generally, any Sasakian space form (see, for example, [3]) is a spherical CRsymmetric pseudo-Hermitian manifold. Indeed, in the Sasakian case, the Webster metric $g$ is CR-symmetric if and only if $M$ is a $\varphi$-symmetric space (for this notion see, for example, [25]).

In the present paper some basic features of CR-symmetric Webster metrics are analyzed (see Theorems 3.2 and 4.4). Our main results can be summerized in the following statement.

THEOREM 1.1. Let $(M, H M, J, \eta)$ be a pseudo-Hermitian manifold of $C R$ dimension $n \geq 2$. Assume that the Webster metric $g:=g_{\eta}$ is not Sasakian. Denote by $\tilde{R}$ the curvature of the Tanaka-Webster connection and by $R$ the Riemannian curvature tensor of $g$. Then:

(a) $(M, H M, J, g)$ is locally CR-symmetric if and only if the underlying contact metric structure $(\varphi, \xi, \eta, g)$ satisfies the $(k, \mu)$-nullity condition - that is, for some $k, \mu \in \mathbb{R}$,

$$
\begin{aligned}
& R(X, Y) \xi=k(\eta(Y) X-\eta(X) Y)+\mu(\eta(Y) h X-\eta(X) h Y), \\
& \text { where } h=\frac{1}{2} \mathcal{L}_{\xi} \varphi .
\end{aligned}
$$

(b) Assume that $g$ is locally CR-symmetric. Then the following are equivalent:

(i) $M$ is spherical;

(ii) the Webster scalar curvature vanishes;

(iii) the pseudoholomorphic sectional curvature $\tilde{K}$ is constant;

(iv) $\mu=2$.

When one of these equivalent conditions holds, then $\tilde{K}=0$ but $\tilde{R} \neq 0$. 
This result provides an interpretation in CR geometry of the theory of contact Riemannian $(k, \mu)$-spaces started in [4] and fully developed in the last decade by several authors, especially by Boeckx in [6-8].

We shall present several applications of Theorem 1.1. First of all, one can obtain a complete classification of the simply connected, spherical, CR-symmetric pseudoHermitian manifolds, as follows. We remark that from the classification of Sasakian $\varphi$-symmetric spaces carried out by Jiménez and Kowalski in [18] for $n \geq 2$ and $0<k<n$ there exists a principal fibre bundle $P_{k}^{n} \rightarrow \mathbb{C P}^{k} \times \mathbb{C H}^{n-k}$ with $\varphi$-symmetric Sasakian total space, the base space $N=\mathbb{C P}^{k} \times \mathbb{C H}^{n-k}$ being the product of two Kähler space forms with holomorphic curvatures 1 and -1 respectively. This CRsymmetric space $P_{k}^{n}$ is spherical since the base manifold is Bochner-flat (see [10]), according to a result of Webster which identifies the Chern-Moser tensor of $P_{k}^{n}$ with the Bochner tensor of $N$ (see [32] or [14]).

We can now state the following theorem.

THEOREM 1.2. Every simply connected, spherical CR-symmetric pseudo-Hermitian manifold of CR-dimension $n \geq 2$ is homothetic to one of the following spaces:

$$
\mathbb{S}^{2 n+1}, H^{2 n+1}, B^{n} \times \mathbb{R}, T_{1} \mathbb{H}^{n+1}, P_{1}^{n}, \ldots, P_{n-1}^{n} .
$$

In this statement, two pseudo-Hermitian manifolds $\left(M_{i}, H M_{i}, J_{i}, \eta_{i}\right), i=1,2$, are called homothetic if there exists a CR-diffeomorphism $f: M_{1} \rightarrow M_{2}$ such that $f^{*} \eta_{2}=\alpha \eta_{1}$ with $\alpha$ a positive constant. The first three spaces are the simply connected Sasakian space forms as described in [3, p. 114]. $H^{2 n+1}$ denotes the Heisenberg group endowed with its standard Webster flat Sasakian structure, while $B^{n} \times \mathbb{R}$ is the product of a Kähler bounded domain in $\mathbb{C}^{n}$ having constant holomorphic negative curvature with the real line, which carries a Sasakian structure with constant $\varphi$ sectional curvature less than -3 . The fourth space is the tangent sphere bundle $T_{1} \mathbb{H}^{n+1}$ of the Riemannian space form of curvature -1 , with its standard CR structure and pseudo-Hermitian structure studied, for example, in [28]. The remaining $n-1$ spaces are the Sasakian $\varphi$-symmetric spaces described above.

We also get the following consequences of the above classification.

COROLLARY 1.3. Up to homothety, the sphere $\mathbb{S}^{2 n+1}$ is the unique simply connected, compact, spherical CR-symmetric pseudo-Hermitian manifold having CR-dimension $n \geq 2$.

COROllary 1.4. A spherical, CR-symmetric pseudo-Hermitian manifold of CRdimension $n \geq 2$, having positive pseudoholomorphic curvature at some point, is compact and is actually a Sasakian pseudo-Hermitian space form.

The notion of pseudoholomorphic sectional curvature is described in detail in Section 2. We also remark that Theorem 1.2 includes the classification of pseudoparallel strongly pseudoconvex CR manifolds with constant pseudoholomorphic sectional curvature obtained by Cho in [13]. These manifolds are precisely $\mathbb{S}^{2 n+1}$, 
$H^{2 n+1}, B^{n} \times \mathbb{R}$ and $T_{1} \mathbb{H}^{n+1}$. The reason lies in the fact that any pseudo-Hermitian manifold with constant pseudoholomorphic sectional curvature must be spherical. Even though this statement should be almost obvious, it is not quoted in the paper of Webster [31] where the Chern-Moser tensor $S$ is first computed by means of pseudoHermitian structures. In [31] some examples of pseudo-Hermitian space forms in $\mathbb{C}^{n}$ are exhibited, and the vanishing of $S$ is verified case by case. Hence we opted to give a general proof, making the necessary computation explicit by using the equivalent Bochner tensor (see Theorem 4.3).

As a further application of Theorem 1.1, in the last section we study the CR geometry of tangent sphere bundles of arbitrary constant radius over Riemannian manifolds with constant curvature. Our approach is slightly more general than that appearing in [30] and [28]. We show that if $M$ is a hyperbolic Riemannian space form, each $T_{r} M$ carries a one-parameter family of CR-symmetric nonhomothetic pseudo-Hermitian structures, exactly one of which is spherical (Theorem 6.2). This should be compared with the relevant fact that a homogeneous CR manifold which is homeomorphic to a sphere admits a unique homogeneous CR structure [17]. Our examples emphasize that 'homeomorphic' cannot be replaced by 'homotopically equivalent', even if the homogeneous CR structure is spherical.

Finally, we mention that in [21] Kowalski and Sekizawa show how the geometric properties of tangent sphere bundles $T_{r} M$ of a given Riemannian manifold $(M, g)$ are influenced by the value of the radius $r$. Since in the constant curvature case the metric induced by $g$ on each $T_{r} M$ is Webster and CR-symmetric, it is natural to ask how the pseudo-Hermitian geometry of $T_{r} M$ varies according to $r$. As regards this problem, applying Theorem 6.2, we obtain partial information given by the following.

COROLlary 1.5. Let $(M, g)$ be a Riemannian manifold of constant curvature $K$ and dimension $n \geq 3$. Consider the standard almost complex structure $J$ on $T M$ defined by

$$
J X^{H}=X^{V}, J X^{V}=-X^{H}, \quad X \in \mathfrak{X}(M),
$$

where $X^{H}$ and $X^{V}$ denote horizontal and vertical lifts.

Endow each $T_{r} M, r>0$, with the induced CR structure and the standard pseudoHermitian structure $\eta_{r}$. Then:

(a) $K<0$ if and only if there exists $r>0$ such that $T_{r} M$ is a spherical CR manifold;

(b) $K>0$ if and only if there exists $r>0$ such that the Webster metric $g_{\eta_{r}}$ is Sasakian;

(c) $K=0$ if and only if $\left(T_{r} M, H\left(T_{r} M\right), J, \eta_{r}\right)$ and $\left(T_{r^{\prime}} M, H\left(T_{r^{\prime}} M\right), J, \eta_{r^{\prime}}\right)$ are locally homothetic pseudo-Hermitian manifolds for each $r, r^{\prime}>0$.

Moreover, if one of the equivalent conditions in (a) holds, there exists a unique $r_{o}$ such that $T_{r_{o}} M$ is spherical, which is related to $K$ by

$$
K=-\frac{1}{r_{o}^{2}} .
$$


When one of the equivalent conditions in (b) holds there exists a unique $r_{o}$ such that $g_{\eta_{r_{o}}}$ is Sasakian, which is related to $K$ by

$$
K=\frac{1}{r_{o}^{2}} .
$$

\section{Preliminaries}

Let $M$ be a connected $\mathcal{C}^{\infty}$ manifold of dimension $2 n+k, n, k \geq 1$. A partial complex structure of CR-dimension $n$ and CR-codimension $k$ is a pair $(H M, J)$ where $H M$ is a smooth real subbundle of the tangent bundle $T M$ having rank $2 n$, and $J$ is a smooth bundle isomorphism $J: H M \rightarrow H M$ such that $J^{2}=-I$. An almost $C R$ structure is a partial complex structure such that

$$
[X, Y]-[J X, J Y] \in \mathcal{D}
$$

for any $X, Y \in \mathcal{D}$, where $\mathcal{D}$ denotes the module of all smooth sections of $H M$. If, in addition, the formal integrability condition

$$
[J X, J Y]-[X, Y]-J([J X, Y]+[X, J Y])=0
$$

is satisfied, $(M, H M, J)$ is termed a $C R$ manifold. In this paper we shall be concerned only with the case where $M$ has CR-codimension 1 (hypersurface type). Assuming (2.1) and that $M$ is orientable, it is known that there exist globally defined nowhere zero 1 -forms $\eta$ such that $\operatorname{Ker}(\eta)=H M$. The corresponding Levi form is defined by

$$
L_{\eta}(X, Y)=-d \eta(X, J Y), \quad X, Y \in \mathcal{D} .
$$

The almost CR structure is said to be nondegenerate if $L_{\eta}$ is nondegenerate for some $\eta$. In this case, the 1 -form $\eta$ is a contact form, in the sense that $\eta \wedge(d \eta)^{n}$ is a volume form on $M$. Moreover, there exists a unique nowhere vanishing globally defined vector field $\xi$ transverse to $H M$ such that

$$
\eta(\xi)=1, \quad d \eta(\xi, X)=0,
$$

for any $X \in \mathfrak{X}(M)$. The second condition is equivalent to $[\xi, \mathcal{D}] \subset \mathcal{D}$ or $\mathcal{L}_{\xi} \eta=0$, where $\mathcal{L}_{\xi}$ denotes the Lie differentiation with respect to $\xi$.

An almost CR structure is said to be strongly pseudoconvex if $L_{\eta}$ is positive definite for some $\eta$. In this case the Levi form can be canonically extended to a Riemannian metric on $M$, called the Webster metric, defined by

$$
g_{\eta}(X, Y)=L_{\eta}(X, Y), \quad g_{\eta}(X, \xi)=0, \quad g_{\eta}(\xi, \xi)=1,
$$

for any $X, Y \in \mathcal{D}$. Such a 1 -form $\eta$ will be called a pseudo-Hermitian structure.

By a pseudo-Hermitian manifold we shall mean a strongly pseudoconvex CR manifold $(M, H M, J, \eta)$ on which a pseudo-Hermitian structure has been fixed. The partial complex structure $J$ of a pseudo-Hermitian manifold can be canonically extended to a tensor field $\varphi$ of type $(1,1)$ on $M$ such that $\varphi(\xi)=0$ and $\varphi X=J X$ for any $X \in \mathcal{D}$, which is an $f$-structure with rank $2 n$. The tensors $\left(\varphi, \xi, \eta, g_{\eta}\right)$ make up a contact metric structure on $M$ in the sense of [3]. Conversely, if $M$ is a contact metric 
manifold with structure $(\varphi, \xi, \eta, g)$, then $M$ admits a strongly pseudoconvex almost CR structure given by $H M=\operatorname{Im}(\varphi)$ and $J=\left.\varphi\right|_{H M}$. The Webster metric $g_{\eta}$ coincides with $g$. Tanno proved that this almost CR structure is a CR structure if and only if

$$
\left(\nabla_{X} \varphi\right) Y=g(X+h X, Y) \xi-\eta(Y)(X+h X)
$$

for any $X, Y \in \mathfrak{X}(M)$ (see [27]). Here $\nabla$ is the Levi-Civita connection of the Webster metric $g$ and $h$ is the symmetric operator $h:=\frac{1}{2} \mathcal{L}_{\xi} \varphi$.

We also recall that in the literature a pseudo-Hermitian manifold $(M, H M, J, \eta)$ such that $h=0$ is called standard or regular while the metric $g_{\eta}$ is called a Sasakian metric. We shall also use the term Sasakian manifold as a synonym for the regular pseudo-Hermitian manifold.

Next we recall a special class of contact metric manifolds with which we will be concerned in the following, the so-called $(k, \mu)$-spaces. Such a space is characterized by the following property of the Riemannian curvature tensor, known in the literature as the $(k, \mu)$-nullity condition:

$$
R(X, Y) \xi=k(\eta(Y) X-\eta(X) Y)+\mu(\eta(Y) h X-\eta(X) h Y), \quad k, \mu \in \mathbb{R},
$$

for any $X, Y \in \mathfrak{X}(M)$. In [4] the authors prove the relation $h^{2}=(k-1) \varphi^{2}$ which implies that $k \leq 1$. If $k=1$, then $h=0$ and $M$ is a Sasakian manifold. If $k<1$, the contact metric structure is not Sasakian and $M$ admits three mutually orthogonal integrable distributions $D(0)=\mathbb{R} \xi, D(\lambda)$ and $D(-\lambda)$, determined by the eigenspaces of $h$, with $\lambda=\sqrt{1-k}$. Moreover, when $k<1$ the curvature tensor is completely determined by the condition (2.3), and its explicit expression is the following [6]:

$$
\begin{aligned}
R(X, Y) Z=(1- & \left.\frac{\mu}{2}\right)(g(Y, Z) X-g(X, Z) Y) \\
& +g(Y, Z) h X-g(X, Z) h Y+g(h Y, Z) X-g(h X, Z) Y \\
& +\frac{1-(\mu / 2)}{1-k}(g(h Y, Z) h X-g(h X, Z) h Y) \\
& -\frac{\mu}{2}(g(\varphi Y, Z) \varphi X-g(\varphi X, Z) \varphi Y)+\mu g(\varphi X, Y) \varphi Z \\
& +\frac{k-(\mu / 2)}{1-k}(g(\varphi h Y, Z) \varphi h X-g(\varphi h X, Z) \varphi h Y) \\
& +\eta(X)\left(\left(k-1+\frac{\mu}{2}\right) g(Y, Z)+(\mu-1) g(h Y, Z)\right) \xi \\
& -\eta(Y)\left(\left(k-1+\frac{\mu}{2}\right) g(X, Z)+(\mu-1) g(h X, Z)\right) \xi \\
& -\eta(X) \eta(Z)\left(\left(k-1+\frac{\mu}{2}\right) Y+(\mu-1) h Y\right) \\
& +\eta(Y) \eta(Z)\left(\left(k-1+\frac{\mu}{2}\right) X+(\mu-1) h X\right) .
\end{aligned}
$$


In [7], Boeckx introduced the invariant

$$
I=\frac{1-\mu / 2}{\sqrt{1-k}}
$$

and proved that two non-Sasakian $(k, \mu)$-spaces are locally homothetic pseudoHermitian manifolds if and only if their invariants coincide.

Moreover, the non-Sasakian $(k, \mu)$-spaces are also characterized by the requirement that

$$
g\left(\left(\nabla_{X} h\right) Y, Z\right)=0
$$

for any $X, Y, Z \in \mathcal{D}$. This fact has been proved in [9], where contact metric structures satisfying (2.5) are called $\eta$-parallel.

We conclude this section by recalling some basic facts about the Tanaka-Webster connection. We shall refer to [23].

THEOREM 2.1. Let $(M, H M, J, \eta)$ be a pseudo-Hermitian manifold with subordinate contact metric structure $(\varphi, \xi, \eta, g)$. There is a unique linear connection $\tilde{\nabla}$ such that

$$
\tilde{\nabla} \varphi=0, \quad \tilde{\nabla} \xi=0, \quad \tilde{\nabla} \eta=0, \quad \tilde{\nabla} g=0, \quad \tilde{T}_{\mathcal{D}}=0, \quad F=-\frac{1}{2} \varphi \mathcal{L}_{\xi} \varphi,
$$

where $\tilde{T}$ is the torsion tensor field of $\tilde{\nabla}, \tilde{T}_{\mathcal{D}}(X, Y)$ denotes the $\mathcal{D}$-component of $\tilde{T}(X, Y)$ for any $X, Y \in \mathcal{D}$, and $F$ is the tensor field of type $(1,1)$ defined by $F X=\tilde{T}(\xi, X)$ for any $X \in \mathfrak{X}(M)$.

The linear connection in the above statement is called the canonical connection or the Tanaka-Webster connection of the pseudo-Hermitian manifold $M$. Denoting by $\nabla$ the Levi-Civita connection of $g$, then $\tilde{\nabla}=\nabla+H$, with

$$
H(X, Y)=g(X, \varphi Y) \xi+\eta(X) \varphi Y+\eta(Y) \varphi X+g(F X, Y) \xi-\eta(Y) F X
$$

for any $X, Y \in \mathfrak{X}(M)$. The curvature tensor $\tilde{R}$ of $\tilde{\nabla}$ satisfies

$$
\tilde{R}(X, Y) \xi=0, \quad \tilde{R}(X, Y) \varphi=\varphi \tilde{R}(X, Y), \quad \tilde{R}(X, Y) \mathcal{D} \subset \mathcal{D},
$$

for any $X, Y \in \mathfrak{X}(M)$.

If $\sigma \subset H_{x} M$ is a holomorphic 2-plane in $x \in M$ (that is, $J \sigma=\sigma$ ), then the quantity

$$
\tilde{K}(\sigma)=\tilde{R}_{x}(X, J X, X, J X),
$$

where $\{X, J X\}$ is an orthonormal basis of $\sigma$, depends only on $\sigma$ and will be called the pseudoholomorphic sectional curvature of $\sigma$. If $\tilde{K}(\sigma)$ does not depend on $\sigma$ and on the point $x$, then $M$ will be called a pseudo-Hermitian space form. 


\section{CR-symmetric Webster metrics}

Let $(M, H M, J, g)$ be a Hermitian almost CR manifold, that is, an almost CR manifold, having CR-codimension $k \geq 1$, on which a Riemannian metric $g$ is fixed, whose restriction to $H M$ is Hermitian with respect to $J$. Denote by $\mathcal{D}_{\infty} \subset \mathfrak{X}(M)$ the Lie algebra generated by $\mathcal{D}$. Let $\sigma: M \rightarrow M$ be an isometric CR-diffeomorphism. Then $\sigma$ is called a symmetry at the point $x \in M$ if $x$ is a fixed point of $\sigma$ and the differential of $\sigma$ at $x$ coincides with - Id on the subspace $\mathcal{D}_{\infty}(x)^{\perp} \oplus H_{x} M$ of $T_{x} M$. Here $\mathcal{D}_{\infty}(x)=\left\{X_{x} \mid X \in \mathcal{D}_{\infty}\right\}$.

A connected Hermitian almost CR manifold $M$ is called (globally) symmetric if for each point $x \in M$ there exists a symmetry $\sigma_{x}$ at $x$ (see [19]). We shall also say that $g$ is a CR-symmetric Hermitian metric on $(M, H M, J)$. Since the symmetry at $x$ is uniquely determined (see [19, Theorem 3.3]) it makes sense also to define Hermitian locally CR-symmetric almost CR spaces in a natural manner. Observe that, since the symmetries are CR maps, for this class of almost CR spaces the integrability condition (2.2) is automatically satisfied.

It is proved in [19] that a Hermitian symmetric CR space $M$ is $C R$-homogeneous: in fact the subgroup of the automorphism group $\operatorname{Aut}_{C R}(M)$ generated by the symmetries acts transitively. In particular, every Hermitian symmetric CR space $M$ is a real analytic CR manifold.

From now on we specialize to strongly pseudoconvex CR manifolds of hypersurface type and discuss CR-symmetric Webster metrics. We remark that for a pseudoHermitian manifold $\mathcal{D}_{\infty}(x)=T_{x} M$, so that a symmetry at a point $x$ is characterized by the condition $(d s)_{x}=-\mathrm{Id}$ on $H_{x} M$.

LemMa 3.1. Let $(M, H M, J, \eta)$ be a pseudo-Hermitian manifold. Denote by $g=g_{\eta}$ the Webster metric associated with $\eta$ and by $(\varphi, \xi, \eta, g)$ the corresponding contact metric structure. Let $x \in M$ and assume that $\sigma_{x}: U \rightarrow U$ is a local symmetry at $x$ defined on an open neighbourhood of $x$. Then $\sigma_{x}$ is local automorphism of $(\varphi, \xi, \eta, g)$.

PROOF. According to [19, Remark 3.4] we see that the differential of $\sigma_{x}$ at $x$ is given by

$$
\left(d \sigma_{x}\right)_{x}=-\mathrm{Id}+2 \eta_{x} \otimes \xi_{x} .
$$

Thus $\left(d \sigma_{x}\right)_{x}\left(\xi_{x}\right)=\xi_{x}$, which implies that $\left(\sigma_{x}\right)_{*} \xi=\xi$ because $\sigma_{x}$ is a CR-isometry. It also follows that $\left(\sigma_{x}\right)^{*} \eta=\eta$ because $\eta$ is dual to $\xi$ with respect to $g$. Since $\sigma_{x}$ is a CR map it follows immediately that it also preserves the tensor field $\varphi$.

At this point we get the following characterization of CR-symmetric Webster metrics.

TheOREM 3.2. Let $(M, H M, J, \eta)$ be a pseudo-Hermitian manifold. Assume that the Webster metric $g_{\eta}$ is not Sasakian. The following conditions are equivalent:

(a) the Webster metric $g_{\eta}$ is locally CR-symmetric;

(b) the underlying contact metric structure satisfies the $(k, \mu)$-nullity condition. 
PROOF. $(a) \Rightarrow(b)$. It suffices to prove that the contact metric structure is $\eta$-parallel. Let $X, Y, Z \in \mathcal{D}$. We need to prove that $g\left(\left(\nabla_{X} h\right) Y, Z\right)=0$. Fix a point $x \in M$ and consider a local symmetry $\sigma_{x}$ at $x$. According to the lemma, $\sigma$ preserves the tensor field $h$ and also its covariant derivative. Hence at $x$ we obtain

$$
g_{x}\left(\left(\nabla_{X_{x}} h\right) Y_{x}, Z_{x}\right)=g_{x}\left(\left(\nabla_{d \sigma_{x}\left(X_{x}\right)} h\right) d \sigma_{x}\left(Y_{x}\right), d \sigma_{x}\left(Z_{x}\right)\right)=-g_{x}\left(\left(\nabla_{X_{x}} h\right) Y_{x}, Z_{x}\right)
$$

and the assertion follows.

$(b) \Rightarrow(a)$. In [6] the following tensor field $T$ is considered:

$$
T_{X} Y=(g(\varphi X, Y)+g(\varphi h X, Y)) \xi-\eta(Y)(\varphi X+\varphi h X)-\frac{\mu}{2} \eta(X) \varphi Y .
$$

$T$ is a homogeneous structure for the contact metric structure $(\varphi, \xi, \eta, g)$, that is,

$$
\begin{gathered}
\bar{\nabla} g=\bar{\nabla} R=\bar{\nabla} T=0, \\
\bar{\nabla} \xi=\bar{\nabla} \eta=\bar{\nabla} \varphi=0,
\end{gathered}
$$

where $\bar{\nabla}=\nabla-T, \nabla$ being the Levi-Civita connection and $R$ its curvature tensor. We also remark that $\bar{\nabla} \bar{R}=\bar{\nabla} \bar{T}=0$, where $\bar{R}$ is the curvature tensor and $\bar{T}$ is the torsion of $\bar{\nabla}$. Fix a point $x \in M$. From the expression for $T$ we see that $T_{x}$ is preserved by the linear transformation $L:=-\mathrm{Id}+2 \eta_{x} \otimes \xi_{x}$ of $T_{x} M$. Moreover, from expression (2.4) for the curvature tensor $R$, it is straightforward to verify that $L$ also preserves $R_{x}$. This in turn implies that $L$ preserves $\bar{R}_{x}$. Hence, by a standard argument (see [20, p. 261]), there exists an affine transformation $\sigma: U \rightarrow U$ with respect to $\bar{\nabla}$, defined on an open neighbourhood of $x$, such that $(d \sigma)_{x}=L$. From the parallelism of the structure tensors $(\varphi, \xi, \eta, g)$, it follows that $\sigma$ is actually a CR-isometry, and thus a local symmetry at $x$.

To end this section, we shall prove that for Sasakian manifolds, local symmetry in the sense of [19] is actually equivalent to a similar concept in the literature, namely locally $\varphi$-symmetric contact metric structure (see $[3,6])$. The latter is defined by the requirement that the characteristic reflections, that is, the reflections with respect to the integral curves of $\xi$, be local isometries. A (global) Sasakian $\varphi$-symmetric space is a Sasakian locally $\varphi$-symmetric space whose characteristic reflections are globally defined, and $\xi$ generates a global one-parameter group of automorphisms of the contact structure [25].

Proposition 3.3. Let $(M, H M, J, \eta)$ be a pseudo-Hermitian manifold. Assume that the Webster metric $g=g_{\eta}$ is Sasakian. The following conditions are equivalent:
(a) $\left(M, H M, J, g_{\eta}\right)$ is a locally (globally) CR-symmetric pseudo-Hermitian manifold;

(b) $(M, \varphi, \xi, \eta, g)$ is a locally (globally) $\varphi$-symmetric space. 
PROOF. We treat the local statement first.

(a) $\Rightarrow$ (b). Since the metric $g_{\eta}$ is Sasakian, it is known that the geodesic reflection $s_{x}$ at a point $x \in M$ with respect to the integral curve of $\xi$ through $x$ is given, on a normal neighbourhood $U$ of $x$, by

$$
s_{x}=\exp _{x} \circ L \circ \exp _{x}^{-1}
$$

where $L=-\mathrm{Id}+2 \eta_{x} \otimes \xi_{x}$ [5]. By (3.1) in Lemma 3.1 the symmetry $\sigma$ at $x$ coincides with $s_{x}$ on a suitable $U^{\prime} \subset U$. Hence $s_{x}$ is a local isometry. This means that $M$ is locally $\varphi$-symmetric.

(b) $\Rightarrow$ (a) Under assumption (b), it is proved in [5] that $s_{x}$ is a local automorphism of the contact metric structure, in particular it is a CR-isometry and $(d s)_{x}=-\mathrm{Id}$ on $H_{x} M$, so that $s_{x}$ is a symmetry at $x$.

Finally, as for the global statement, we remark that if $g_{\eta}$ is globally CRsymmetric, $M$ is CR-homogeneous and Riemannian homogeneous, hence $\xi$ is complete, being a Killing field.

\section{The Bochner-type tensor of a symmetric CR manifold}

Let $(M, H M, J)$ be a strongly pseudoconvex CR manifold having CR-dimension $n \geq 2$ and let $\eta$ and $\eta^{\prime}$ be two pseudo-Hermitian structures, with subordinate contact metric structures $(\varphi, \xi, \eta, g)$ and $\left(\varphi^{\prime}, \xi^{\prime}, \eta^{\prime}, g^{\prime}\right)$. As proved in [23], these structures are related by

$$
\begin{gathered}
\eta^{\prime}=e^{2 \mu} \eta, \quad \xi^{\prime}=e^{-2 \mu}(\xi+Q), \quad \varphi^{\prime}=\varphi+\eta \otimes P, \\
g^{\prime}(X, Y)=e^{2 \mu} g(X, Y), \quad \forall X, Y \in \mathcal{D},
\end{gathered}
$$

where $\mu$ is a $\mathcal{C}^{\infty}$-function, and $P \in \mathcal{D}$ is defined by $g(P, X)=d \mu(X)$ for $X \in \mathcal{D}$ and $Q=J P$. In [24] the authors derive a pseudoconformal invariant on the CR manifold, that is, an invariant of the change (4.1), called the Bochner curvature tensor. A more general treatment is given in [29], where almost CR manifolds are allowed. There it is proved that for CR manifolds this tensor coincides with the Chern-MoserTanaka invariant $[12,26]$. The definition of the Bochner curvature tensor involves the curvature of the canonical connection $\tilde{\nabla}$, as described in the following.

As usual, the Ricci tensor field $s$ of $\tilde{\nabla}$ is defined by

$$
s(X, Y)=\operatorname{tr}(V \rightarrow \tilde{R}(V, X) Y)
$$

for any $X, Y \in \mathfrak{X}(M)$. One can define another Ricci tensor field $k$ by

$$
k(X, Y)=\frac{1}{2} \operatorname{tr}(\varphi \tilde{R}(X, \varphi Y))
$$

for any $X, Y \in \mathfrak{X}(M)$. Both $s$ and $k$ are symmetric when restricted to $\mathcal{D}$, and they satisfy

$$
k(X, Y)=s(X, Y)+2(n-1) g(F J X, Y)
$$


for any $X, Y \in \mathcal{D}$. We recall (see Theorem 2.1) that the tensor $F$ is given by $F=$ $-\varphi \circ h$. We shall also denote by $\rho$ the Webster scalar curvature which is defined by

$$
\rho=\operatorname{tr}(s)
$$

The expression for the Bochner curvature tensor also involves the tensors $l$ and $m$ defined by

$$
\begin{aligned}
l(X, Y) & =-\frac{1}{2(n+2)} k(X, Y)+\frac{1}{8(n+1)(n+2)} \rho g(X, Y), \\
m(X, Y) & =l(J X, Y),
\end{aligned}
$$

for any $X, Y \in \mathcal{D}$, and the tensors $L$ and $M$ such that

$$
g(L X, Y)=l(X, Y), \quad g(M X, Y)=m(X, Y),
$$

which satisfy $L J=J L=M$. Then the Bochner curvature tensor is defined by

$$
B=B_{0}+B_{1}
$$

where, for any $X, Y, Z \in \mathcal{D}$,

$$
\begin{gathered}
B_{0}(X, Y) Z=\tilde{R}(X, Y) Z-2\{m(X, Y) J Z+g(J X, Y) M Z\}+l(Y, Z) X \\
-l(X, Z) Y+m(Y, Z) J X-m(X, Z) J Y+g(Y, Z) L X \\
\quad-g(X, Z) L Y+g(J Y, Z) M X-g(J X, Z) M Y, \\
B_{1}(X, Y) Z=\frac{1}{2}\{\tilde{R}(J X, J Y) Z-\tilde{R}(X, Y) Z\} .
\end{gathered}
$$

REMARK 4.1. In [23] and [24] the authors actually consider the canonical connection associated with the structure $(\bar{\varphi}, \bar{\xi}, \bar{\eta}, \bar{g})$ such that

$$
\bar{\varphi}=-\varphi, \quad \bar{\xi}=\frac{1}{2} \xi, \quad \bar{\eta}=2 \eta, \quad \bar{g}=4 g .
$$

It can easily be seen that the connections associated with $(\bar{\varphi}, \bar{\xi}, \bar{\eta}, \bar{g})$ and with $(\varphi, \xi, \eta, g)$ through conditions in (2.6) coincide. Since our computations involve $(k, \mu)$-spaces, we prefer to express the Bochner curvature tensor in terms of $(\varphi, \xi, \eta, g)$.

LEMMA 4.2. Let $(M, H M, J, \eta)$ be a pseudo-Hermitian manifold with associated contact metric structure $(\varphi, \xi, \eta, g)$. Let $\nabla$ be the Levi-Civita connection of $g$ and $\tilde{\nabla}$ the canonical connection. Then the corresponding curvature tensors $R$ and $\tilde{R}$ are related by:

$$
\begin{aligned}
\tilde{R}(X, Y) Z=R(X, & Y) Z+g(F Y-\varphi Y, Z)(F X-\varphi X) \\
& -g(F X-\varphi X, Z)(F Y-\varphi Y)-2 g(\varphi X, Y) \varphi Z \\
& +g\left(\left(\tilde{\nabla}_{X} F\right) Y-\left(\tilde{\nabla}_{Y} F\right) X, Z\right) \xi
\end{aligned}
$$


for any $X, Y, Z \in \mathcal{D}$. Denoting by Ric the Ricci tensor of $g$, then the Ricci tensor $s$ satisfies

$$
s(X, Y)=\operatorname{Ric}(X, Y)-g(R(X, \xi) \xi, Y)-g\left(F^{2} X, Y\right)+3 g(X, Y)
$$

for any $X, Y \in \mathcal{D}$. Finally, denoting by $\tau$ the scalar curvature of $g$, the Webster scalar curvature is given by

$$
\rho=\tau-2 \operatorname{Ric}(\xi, \xi)-\operatorname{tr}\left(F^{2}\right)+6 n .
$$

PROOF. Straightforward computations using (2.7) and the parallelism of the structure tensors with respect to $\tilde{\nabla}$.

THEOREM 4.3. A pseudo-Hermitian space form of CR-dimension $n \geq 2$ is a spherical CR manifold.

Proof. Consider a pseudo-Hermitian space form $(M, H M, J, \eta)$. We need to prove that the Bochner curvature tensor vanishes. Since the pseudoholomorphic sectional curvature is a constant $c$, by [13, Proposition 5.2], using (4.5) we obtain the following formula for the curvature tensor of the canonical connection $\tilde{\nabla}$ :

$$
\begin{aligned}
\tilde{R}(X, Y) Z=\frac{c}{4}\{ & g(Y, Z) X-g(X, Z) Y \\
& +g(\varphi Y, Z) \varphi X-g(\varphi X, Z) \varphi Y+2 g(X, \varphi Y) \varphi Z\} \\
& +g(h Y, Z) X-g(h X, Z) Y+g(\varphi h Y, Z) \varphi X-g(\varphi h X, Z) \varphi Y \\
& +g(Y, Z) h X-g(X, Z) h Y+g(\varphi Y, Z) \varphi h X-g(\varphi X, Z) \varphi h Y
\end{aligned}
$$

for any $X, Y, Z \in \mathcal{D}$. Using (4.4), a straightforward computation shows that

$$
\begin{aligned}
& B_{1}(X, Y) Z=g(h X, Z) Y-g(h Y, Z) X+g(\varphi h X, Z) \varphi Y-g(\varphi h Y, Z) \varphi X \\
&+g(X, Z) h Y-g(Y, Z) h X+g(\varphi X, Z) \varphi h Y-g(\varphi Y, Z) \varphi h X .
\end{aligned}
$$

Now, taking $X, Y \in \mathcal{D}$, observing that $g(\tilde{R}(\xi, X) Y, \xi)=0$ and $\operatorname{tr}(h)=\operatorname{tr}(h \varphi)=0$, for the Ricci tensor field $s$ we obtain

$$
s(X, Y)=\frac{c}{2}(n+1) g(X, Y)+2(n-1) g(h X, Y) .
$$

Applying (4.2), since $F=h \varphi$, for the Ricci tensor field $k$ we obtain

$$
k(X, Y)=\frac{c}{2}(n+1) g(X, Y) .
$$

Computing the Webster scalar curvature gives

$$
\rho=\operatorname{cn}(n+1) \text {. }
$$


With these ingredients one can compute the tensor fields $l, m, L$ and $M$ which are given by

$$
\begin{gathered}
l(X, Y)=-\frac{c}{8} g(X, Y), \quad m(X, Y)=-\frac{c}{8} g(\varphi X, Y), \\
L X=-\frac{c}{8} X, \quad M X=-\frac{c}{8} \varphi X .
\end{gathered}
$$

Applying (4.3) leads to

$$
\begin{aligned}
& B_{0}(X, Y) Z=g(h Y, Z) X-g(h X, Z) Y+g(\varphi h Y, Z) \varphi X-g(\varphi h X, Z) \varphi Y \\
&+ g(Y, Z) h X-g(X, Z) h Y+g(\varphi Y, Z) \varphi h X-g(\varphi X, Z) \varphi h Y \\
&+\frac{c}{4}\{g(Y, Z) X-g(X, Z) Y+g(\varphi Y, Z) \varphi X-g(\varphi X, Z) \varphi Y \\
&+2 g(X, \varphi Y) \varphi Z\}+\frac{c}{2} g(\varphi X, Y) \varphi Z \\
&-\frac{c}{4}\{g(Y, Z) X-g(X, Z) Y+g(\varphi Y, Z) \varphi X-g(\varphi X, Z) \varphi Y\} \\
&= g(h Y, Z) X-g(h X, Z) Y+g(\varphi h Y, Z) \varphi X-g(\varphi h X, Z) \varphi Y \\
&+g(Y, Z) h X-g(X, Z) h Y+g(\varphi Y, Z) \varphi h X-g(\varphi X, Z) \varphi h Y .
\end{aligned}
$$

It follows that $B=B_{0}+B_{1}=0$.

THEOREM 4.4. Let $(M, H M, J, \eta)$ be a non-Sasakian locally CR-symmetric pseudoHermitian manifold having $C R$-dimension $n \geq 2$. Let $(\varphi, \xi, \eta, g)$ be the underlying contact metric structure. Then the Bochner curvature tensor is given by

$$
\begin{aligned}
B(X, Y) Z=\frac{\rho}{4 n^{2}(n+1)}(g(Y, Z) X-g(X, Z) Y & \\
& +g(\varphi Y, Z) \varphi X-g(\varphi X, Z) \varphi Y-2 g(\varphi X, Y) \varphi Z) \\
& +\frac{\rho}{2 n \operatorname{tr}\left(h^{2}\right)}(g(h Y, Z) h X-g(h X, Z) h Y \\
& +g(\varphi h Y, Z) \varphi h X-g(\varphi h X, Z) \varphi h Y)
\end{aligned}
$$

for any $X, Y, Z \in \mathcal{D}$. Moreover, the following conditions are equivalent:

(i) $B=0$;

(ii) the Boeckx invariant $I=0$;

(iii) the Webster scalar curvature $\rho$ vanishes;

(iv) $M$ has constant pseudoholomorphic curvature.

If any of the above conditions holds, then $\tilde{K}=0$, but $\tilde{R} \neq 0$.

PROOF. First we compute the curvature $\tilde{R}$ of the canonical connection $\tilde{\nabla}$. Let us consider $X, Y, Z \in \mathcal{D}$. We remark that since $M$ is a $(k, \mu)$-space, then $R(X, Y) Z \in \mathcal{D}$. Hence from (4.5) we obtain

$$
\begin{aligned}
\tilde{R}(X, Y) Z=R(X, Y) Z+g(\varphi h Y+\varphi Y, Z)(\varphi h X+\varphi X) & \\
& -g(\varphi h X+\varphi X, Z)(\varphi h Y+\varphi Y)-2 g(\varphi X, Y) \varphi Z,
\end{aligned}
$$


using $F=-\varphi h$. Hence, taking into account (2.4), we get

$$
\begin{aligned}
\tilde{R}(X, Y) Z=(1- & \left.\frac{\mu}{2}\right)(g(Y, Z) X-g(X, Z) Y \\
& +g(\varphi Y, Z) \varphi X-g(\varphi X, Z) \varphi Y-2 g(\varphi X, Y) \varphi Z) \\
& +\frac{1-(\mu / 2)}{1-k}(g(h Y, Z) h X-g(h X, Z) h Y \\
& +g(\varphi h Y, Z) \varphi h X-g(\varphi h X, Z) \varphi h Y) \\
& +g(Y, Z) h X-g(X, Z) h Y+g(h Y, Z) X-g(h X, Z) Y \\
& +g(\varphi h Y, Z) \varphi X-g(\varphi h X, Z) \varphi Y \\
& +g(\varphi Y, Z) \varphi h X-g(\varphi X, Z) \varphi h Y .
\end{aligned}
$$

Computing $\tilde{R}(\varphi X, \varphi Y) Z$, from (4.4) we get

$$
\begin{aligned}
B_{1}(X, Y) Z=-g(Y, Z) h X+g(X, Z) h Y+g(\varphi Y, Z) h \varphi X-g(\varphi X, Z) h \varphi Y \\
-g(h Y, Z) X+g(h X, Z) Y+g(h \varphi Y, Z) \varphi X-g(h \varphi X, Z) \varphi Y .
\end{aligned}
$$

The Riemannian Ricci tensor is given by (see [4])

$$
\operatorname{Ric}(X, Y)=(2(n-1)-n \mu) g(X, Y)+(2(n-1)+\mu) g(h X, Y)
$$

for any $X, Y \in \mathcal{D}$. Using (4.6), since $F^{2} X=h^{2} X=(1-k) X$, we get

$$
s(X, Y)=n(2-\mu) g(X, Y)+2(n-1) g(h X, Y),
$$

and the Webster scalar curvature is

$$
\rho=2 n^{2}(2-\mu)
$$

which proves that $I$ vanishes if and only if $\rho=0$. Applying (4.2),

$$
k(X, Y)=n(2-\mu) g(X, Y) .
$$

With these elements, the tensors $l, m, L, M$ are given by

$$
\begin{gathered}
l(X, Y)=\frac{n(\mu-2)}{4(n+1)} g(X, Y), \quad m(X, Y)=\frac{n(\mu-2)}{4(n+1)} g(\varphi X, Y), \\
L X=\frac{n(\mu-2)}{4(n+1)} X,
\end{gathered}
$$

Using (4.3) and the expression for $\tilde{R}$, a straightforward computation shows that

$$
\begin{aligned}
B_{0}(X, Y) Z=\frac{2-}{2(n+} & (g(Y, Z) X-g(X, Z) Y \\
& +g(\varphi Y, Z) \varphi X-g(\varphi X, Z) \varphi Y-2 g(\varphi X, Y) \varphi Z) \\
& +\frac{2-\mu}{2(1-k)}(g(h Y, Z) h X-g(h X, Z) h Y \\
& +g(\varphi h Y, Z) \varphi h X-g(\varphi h X, Z) \varphi h Y)
\end{aligned}
$$




$$
\begin{aligned}
& +g(Y, Z) h X-g(X, Z) h Y-g(\varphi Y, Z) h \varphi X+g(\varphi X, Z) h \varphi Y \\
& +g(h Y, Z) X-g(h X, Z) Y-g(h \varphi Y, Z) \varphi X+g(h \varphi X, Z) \varphi Y,
\end{aligned}
$$

and we get the expression (4.8) for $B=B_{0}+B_{1}$, since $\operatorname{tr}\left(h^{2}\right)=2 n(1-k)$. The equivalence of (i) and (iii) is an immediate consequence. As for the pseudoholomorphic sectional curvature, consider a holomorphic 2-plane $\sigma=\langle X, J X\rangle$ where $X$ is a unit holomorphic tangent vector at some point $x \in M$. Using the expression for $\tilde{R}$, we get

$$
\begin{aligned}
\tilde{K}(\sigma) & =g(\tilde{R}(X, \varphi X) \varphi X, X) \\
& =2(2-\mu)-\frac{2-\mu}{1-k}\left(g(h X, X)^{2}+g(h \varphi X, X)^{2}\right),
\end{aligned}
$$

showing that $\tilde{K}$ vanishes for $\mu=2$. Conversely, if $\tilde{K}$ is constant, Theorem 4.3 guarantees that $B=0$.

\section{Classification result}

In this section we prove Theorem 1.2 and its corollaries.

Proof of TheOREM 1.2. Let $(M, H M, J, \eta)$ be a simply connected pseudoHermitian manifold which is a spherical CR manifold and such that $g_{\eta}$ is CRsymmetric. If $g_{\eta}$ is not Sasakian, according to Theorem $4.4, M$ is a $(k, \mu)$-space with vanishing Boeckx invariant. It follows that $M$ is homothetic to $T_{1} \mathbb{H}^{n+1}$ endowed with its standard CR structure, since it is known that the Boeckx invariant of $T_{1} \mathbb{H}^{n+1}$ vanishes [4]. Next we consider the case where $g_{\eta}$ is Sasakian. Then $M$ is a simply connected Sasakian $\varphi$-symmetric space and, according to [18] or [25, Theorem 6.1], it is a principal fibre bundle $\pi: M \rightarrow N$ over a simply connected Hermitian globally symmetric space $\left(N, g_{o}\right)$ and $\pi:\left(M, g_{\eta}\right) \rightarrow\left(N, g_{o}\right)$ is a Riemannian submersion with fibres tangent to $\xi$, which is also a CR map. Moreover, since $M$ is spherical, $N$ is Bochner-flat. Indeed, denoting by $B_{N}$ the Bochner tensor of $N$, by a result of Webster [32] already quoted in the Introduction,

$$
\pi_{*}(B(X, Y) Z)=B_{N}\left(\pi_{*} X, \pi_{*} Y\right) \pi_{*} Z
$$

for any $x \in M$ and $X, Y, Z \in H_{x} M$. Now, according to a result of Matsumoto and Tanno (see [22] or [10, Proposition 2.5]) $N$ is either a simply connected Kähler space form or is isometric to a product $N^{k}(c) \times N^{n-k}(-c), c>0$, of two simply connected Kähler space forms with holomorphic curvatures respectively $c$ and $-c$. In the first case, $M$ is a Sasakian space form and hence, as a pseudo-Hermitian manifold, it is homothetic to $\mathbb{S}^{2 n+1}, H^{2 n+1}$, or $B^{n} \times \mathbb{R}$. In the last case, up to a homothetic change of the metric $g_{o}$, we can assume $c=1$, yielding a homothetic change of the pseudoHermitian structure $\eta$ of $M$ which turns $M$ into a Sasakian manifold equivalent to the Sasakian $\varphi$-symmetric space $P_{k}^{n}$ over $\mathbb{C P}^{k} \times \mathbb{C H}^{n-k}$.

The proof of Corollary 1.3 is immediate. 
Proof of Corollary 1.4. According to Theorem 4.4, the assumption on $\tilde{K}$ forces $g_{\eta}$ to be Sasakian, hence $M$ is a Sasakian $\varphi$-symmetric space. Thus the simply connected covering $\tilde{M}$ is also a $\varphi$-symmetric space which is locally equivalent to $M$ as a pseudo-Hermitian manifold. In particular, $\tilde{M}$ is a spherical symmetric CR space with positive pseudoholomorphic curvature at some point, which is a principal fibre bundle $\pi: \tilde{M} \rightarrow N$ over a Hermitian globally symmetric space $N$. Now, comparing with the classification in Theorem 1.2, $\tilde{M}$ cannot be homothetic to any of the spaces $P_{k}^{n}$. Indeed, observe that, at each point $x$ of $P_{k}^{n}, \tilde{K}(\sigma)=0$ for some holomorphic 2plane $\sigma$. Indeed, choose a holomorphic 2-plane $\sigma^{\prime}$ of $\mathbb{C P}^{k} \times \mathbb{C} \mathbb{H}^{n-k}$ at $\pi(x)$ with vanishing holomorphic curvature. Such a $\sigma^{\prime}$ exists since $\mathbb{C P}^{k}$ and $\mathbb{C H}^{n-k}$ have opposite holomorphic curvatures. Now take $\sigma$ such that $\pi_{*}(\sigma)=\sigma^{\prime}$. For the other models in the classification except for $\mathbb{S}^{2 n+1}$, it is known that at each point $\tilde{K} \leq 0$. Hence $\tilde{M}$ is compact and the assertion follows.

\section{Examples: CR geometry of tangent sphere bundles}

Let $(M, g)$ be a Riemannian manifold of dimension $n \geq 3$. For each $r>0$ we shall denote by $T_{r} M$ the tangent sphere bundle of radius $r$, which is the hypersurface of the tangent bundle $T M$ defined by

$$
T_{r} M=\left\{(x, u) \in T M \mid g_{x}(u, u)=r^{2}\right\}
$$

Here and in the following we consider a point of the tangent bundle $T M$ as a pair $(x, u)$ with $x \in M$ and $u \in T_{x} M$. Let $\pi: T M \rightarrow M$ be the canonical projection such that $\pi(x, u)=x$. For each smooth vector field $X \in \mathfrak{X}(M)$ we shall denote by $X^{V}$ its vertical lift to $T M$ and by $X^{H}$ its horizontal lift with respect to the LeviCivita connection $D$ of $(M, g)$. For details, our standard reference is [3, Ch. 9]. If $t=(x, u)$ is a fixed point of $T M$ and $X \in T_{x} M$, we shall also denote by $X_{t}^{H} \in T_{t} T M$ its horizontal lift and by $X_{t}^{V} \in T_{t} T M$ its vertical lift. Then at each point $t=(x, u)$ of $T_{r} M$ the tangent space to $T_{r} M$ at $t$ is given by

$$
T_{t}\left(T_{r} M\right)=\left\{X_{t}^{H}+Y_{t}^{V} \mid X, Y \in T_{x} M, g_{x}(Y, u)=0\right\} .
$$

Let $\lambda \neq 0$ be a fixed real number. One can define an almost complex structure $J_{\lambda}: T T M \rightarrow T T M$ by

$$
J_{\lambda}\left(X^{H}\right)=\lambda X^{V}, \quad J_{\lambda}\left(X^{V}\right)=-\frac{1}{\lambda} X^{H} .
$$

Since $T_{r} M$ is a real hypersurface of $T M$, it inherits canonically a partial complex structure $\left(H\left(T_{r} M\right), J_{\lambda}\right)$ from $J_{\lambda}$. The holomorphic tangent bundle $H\left(T_{r} M\right)$ can be described as follows. At a fixed point $t=(x, u) \in T_{r} M$,

$$
H_{t}\left(T_{r} M\right)=\left\{X_{t}^{H}+Y_{t}^{V} \mid X, Y \in T_{x} M, g_{x}(X, u)=g_{x}(Y, u)=0\right\} .
$$


We define a global horizontal vector field $\xi \in \mathfrak{X}\left(T_{r} M\right)$ by

$$
\xi_{t}=\frac{2}{\lambda} u_{t}^{H}, \quad t=(x, u) .
$$

Denoting by $\mathfrak{U}$ the canonical vertical vector field of $T M$ (see [3, p. 142] or [21, p. 210]), then

$$
\xi=-2 J_{\lambda} \mathfrak{U}
$$

We recall that the local expression of $\mathfrak{U}$ in a coordinate system $\left(x^{i}, v^{i}\right)$ of $T M$ induced by a local chart $\left(U, x^{1}, \ldots, x^{n}\right)$ of $M$ is

$$
\mathfrak{U}=v^{i} \frac{\partial}{\partial v^{i}} .
$$

Then $\xi$ is everywhere transverse to the holomorphic tangent bundle $H\left(T_{r} M\right)$.

THEOREM 6.1. For each $r>0$ and $\lambda>0,\left(T_{r} M, H\left(T_{r} M\right), J_{\lambda}\right)$ is a strictly pseudoconvex almost $C R$ manifold. Moreover, if $(M, g)$ has constant curvature then $T_{r} M$ is locally CR-symmetric with respect to the Webster metric $g_{\eta_{\lambda}}$, where $\eta_{\lambda}$ is the pseudo-Hermitian structure such that $\eta_{\lambda}(\xi)=1$. If, in addition, $M$ is simply connected and complete, $g_{\eta_{\lambda}}$ is globally CR-symmetric.

PROOF. Define the 1-form $\eta_{\lambda}$ on $T_{r} M$ by

$$
\eta_{\lambda}\left(H\left(T_{r} M\right)\right)=0, \quad \eta_{\lambda}(\xi)=1 .
$$

First we shall prove that the partial complex structure $\left(H\left(T_{r} M\right), J_{\lambda}\right)$ satisfies $(2.1)$ and that the Levi form associated with $\eta_{\lambda}$ is positive definite at each point $t=(x, u) \in$ $T_{r} M$. In order to simplify the notation, in the following we shall denote simply by $J$ both the almost complex structure $J_{\lambda}$ on $T M$ and the partial complex structure induced on $T_{r} M$. We shall also denote $\eta_{\lambda}$ by $\eta$.

Hence we shall verify that, for each $t=(x, u) \in T_{r} M$,

$$
d \eta(Z, W)=d \eta(J Z, J W), \quad d \eta(J Z, Z)>0
$$

where $Z, W \in H_{t}\left(T_{r} M\right)$. To this end, we shall use the fact that, according to (6.2), $H_{t}\left(T_{r} M\right)$ is spanned by vectors of the form $X_{t}^{H}$ and $X_{t}^{V}$ where $X \in \mathfrak{X}(M)$ is such that $X_{x}$ is orthogonal to $u$ with respect to $g$. We remark that $X_{t}^{V}$ can be extended to a global section $X^{t}$ of $H\left(T_{r} M\right)$ defined as follows. Let $g^{S}$ be the Sasaki metric on $T M$ naturally constructed from $g$ (see, for example, [3] or [21]). Observe that $\xi$ is orthogonal to $H\left(T_{r} M\right)$ with respect to the Riemannian metric induced by $g S$ on $T_{r} M$, which will be denoted by the same symbol. Then we set

$$
X^{t}:=X^{V}-\frac{1}{r^{2}} g^{S}\left(X^{V}, \mathfrak{U}\right) \mathfrak{U} .
$$

The vector field $X^{t}$ is the tangential lift of $X$ as defined, for example, in [21, p. 211]. 
We also remark that $X_{t}^{H}$ can be extended to a global section $X^{0}$ of $H\left(T_{r} M\right)$ defined by

$$
X^{0}:=X^{H}-\frac{\lambda}{2 r^{2}} g^{S}\left(X^{V}, \mathfrak{U}\right) \xi .
$$

Actually, according to (6.1),

$$
J X^{0}=\lambda X^{t}, \quad J X^{t}=-\frac{1}{\lambda} X^{0} .
$$

Now we compute $d \eta\left(J X_{t}^{H}, Y_{t}^{H}\right)$ where $X, Y \in \mathfrak{X}(M)$ with $g_{x}\left(X_{x}, u\right)=$ $g_{x}\left(Y_{x}, u\right)=0$. According to (6.6),

$$
2 d \eta\left(J X^{0}, Y^{0}\right)=-\lambda \eta\left[X^{t}, Y^{0}\right] .
$$

Moreover, taking into account the formula (see [3, p. 138])

$$
\left[X^{H}, Y^{V}\right]=\left(D_{X} Y\right)^{V},
$$

we obtain, evaluating at the point $t$,

$$
\eta\left[X^{t}, Y^{0}\right](t)=\eta\left(-\frac{\lambda}{2 r^{2}} X^{V} g^{S}\left(Y^{V}, \mathfrak{U}\right) \xi\right)(t) .
$$

On the other hand, it is readily verified that at $t$ the function $X^{V} g^{S}\left(Y^{V}, \mathfrak{U}\right)$ takes the value $g_{x}(X, Y)$. Hence

$$
\eta\left[X^{t}, Y^{0}\right](t)=-\frac{\lambda}{2 r^{2}} g_{x}(X, Y)
$$

and we conclude that

$$
d \eta\left(J X_{t}^{H}, Y_{t}^{H}\right)=\frac{\lambda^{2}}{4 r^{2}} g_{x}(X, Y) .
$$

Next we compute $d \eta\left(J X_{t}^{V}, Y_{t}^{V}\right)$. Using (6.6) again,

$$
2 d \eta\left(J X^{t}, Y^{t}\right)=\frac{1}{\lambda} \eta\left[X^{0}, Y^{t}\right]
$$

Hence, evaluating at $t$ and taking into account (6.8), we get

$$
d \eta\left(J X_{t}^{V}, Y_{t}^{V}\right)=\frac{1}{4 r^{2}} g_{x}(X, Y) .
$$

Next observe that from

$$
2 d \eta\left(J X^{0}, Y^{t}\right)=-\lambda \eta\left[X^{t}, Y^{t}\right],
$$

using $\left[X^{V}, Y^{V}\right]=0$, we obtain

$$
d \eta\left(J X_{t}^{H}, Y_{t}^{V}\right)=0 .
$$

Thus, taking into account (6.2), equations (6.9), (6.10) and (6.11) yield (6.4). The first assertion is proved. 
Now suppose that $(M, g)$ has constant curvature $K$. We shall compute first the expression for the Webster metric $g_{\eta}$. First of all we claim that $\xi$ is actually the Reeb vector field of $\eta$, that is, $d \eta(Z, \xi)=0$ for any $Z \in \mathfrak{X}\left(T_{r} M\right)$. To justify this, it suffices to verify that

$$
d \eta\left(X^{0}, \xi\right)=d \eta\left(X^{t}, \xi\right)=0
$$

for any $X \in \mathfrak{X}(M)$, or equivalently $\eta\left[X^{0}, \xi\right]=\eta\left[X^{t}, \xi\right]=0$, which in turn is equivalent to

$$
g^{S}\left(\left[X^{0}, \xi\right], \xi\right)=g^{S}\left(\left[X^{t}, \xi\right], \xi\right)=0 .
$$

Indeed, we have

$$
\left[X^{0}, \xi\right]=\left[X^{H}, \xi\right]+\frac{\lambda}{2 r^{2}} \xi g^{S}\left(X^{V}, \mathfrak{U}\right) \xi .
$$

Now fix a point $t=(x, u)$ and consider a coordinate neighbourhood $\left(x^{i}, v^{i}\right)$ around $t$; then we have the local expression $\xi=(2 / \lambda) v^{k}\left(\partial_{k}\right)^{H}$. Assuming $X=X^{i} \partial_{i}, u=$ $u^{k}\left(\partial_{k}\right)_{x}$, we compute

$$
\begin{aligned}
{\left[X^{H}, \xi\right]_{t} } & =\frac{2}{\lambda}\left\{X_{t}^{H}\left(v^{k}\right)\left(\partial_{k}\right)_{t}^{H}+u^{k}\left[X, \partial_{k}\right]_{t}^{H}-\left(R_{x}(X, u) u\right)_{t}^{V}\right\} \\
& =\frac{2}{\lambda}\left\{X_{t}^{H}\left(v^{k}\right)\left(\partial_{k}\right)_{t}^{H}+u^{k}\left(D_{X} \partial_{k}\right)_{t}^{H}-u^{k}\left(D_{\partial_{k}} X\right)_{t}^{H}-\left(R_{x}(X, u) u\right)_{t}^{V}\right\} \\
& =-\frac{2}{\lambda}\left\{u^{k}\left(D_{\partial_{k}} X\right)_{t}^{H}+\left(R_{x}(X, u) u\right)_{t}^{V}\right\}
\end{aligned}
$$

where we have used the formula for the Lie brackets of type $\left[X^{H}, Y^{H}\right]$ in [3, p. 138]. Here $R$ denotes the curvature tensor field of $(M, g)$. It follows that

$$
g^{S}\left(\left[X^{H}, \xi\right], \xi\right)(t)=-\frac{2}{\lambda} u^{k} g^{S}\left(\left(D_{\partial_{k}} X\right)^{H}, \xi\right)(t) .
$$

On the other hand,

$$
g^{S}\left(\frac{\lambda}{2 r^{2}} \xi g^{S}\left(X^{V}, \mathfrak{U}\right) \xi, \xi\right)=\frac{2}{\lambda} \xi g^{S}\left(X^{V}, \mathfrak{U}\right) .
$$

Now, taking into account that, with respect to the Levi-Civita connection $\nabla$ of $\left(T M, g^{S}\right)$, the equality $\nabla_{\partial_{k}^{H}} \mathfrak{U}=0$ holds and that the vertical component of $\nabla_{\partial_{k}^{H}} X^{V}$ is $\left(D_{\partial_{k}} X\right)^{V}$ (see [21, p. 210]), we obtain

$$
\xi g^{S}\left(X^{V}, \mathfrak{U}\right)(t)=\frac{2}{\lambda} u^{k} g^{S}\left(\left(D_{\partial_{k}} X\right)^{V}, \mathfrak{U}\right)(t)=u^{k} g^{S}\left(\left(D_{\partial_{k}} X\right)^{H}, \xi\right)(t) .
$$

Thus, comparing with (6.14), we can conclude that $g^{S}\left(\left[X^{0}, \xi\right], \xi\right)=0$. The proof of $g^{S}\left(\left[X^{t}, \xi\right], \xi\right)=0$ is similar and hence omitted for the sake of brevity.

Now we see that the Webster metric $g_{\eta}$ is the restriction to $T_{r} M$ of the $g$-natural metric on $T M$ :

$$
G=\frac{1}{4 r^{2}} g^{S}+\frac{\lambda^{2}-1}{4 r^{2}} g^{v}
$$


(see $[1,2]$ for the general theory of $g$-natural metrics on tangent bundles). Here $g^{v}$ denotes the vertical lift of $g$ determined by

$$
g^{v}\left(X^{H}, Y^{H}\right)=g(X, Y), g^{v}\left(X^{H}, Y^{V}\right)=g^{v}\left(X^{V}, Y^{V}\right)=0, \quad X, Y \in \mathfrak{X}(M) .
$$

This follows from formulas (6.9), (6.10), and (6.11) for the Levi form at a generic point $t=(x, u) \in T_{r} M$, from the fact that $G\left(X_{t}^{H}, \xi_{t}\right)=G\left(X_{t}^{V}, \xi_{t}\right)=0$ for every $X \in T_{x} M$ with $g_{x}(X, u)=0$, and finally observing that $G(\xi, \xi)=1$.

Now we show that at each point $t=(x, u)$ there exists a local symmetry of $T_{r} M$. Since $(M, g)$ has constant curvature, there exists a local isometry $f: U \rightarrow U$ defined on an open neighbourhood of $x$ in $M$ such that

$$
f(x)=x, d f_{x}(u)=u, d f_{x}(X)=-X \quad \text { if } g_{x}(X, u)=0 .
$$

Indeed, the linear mapping - Id $+\left(2 / r^{2}\right) u^{\mathrm{b}} \otimes u$ of $\left(T_{x} M, g_{x}\right)$ preserves the curvature tensor $R_{x}$. Now consider the induced mapping $F=d f: T U \rightarrow T U$. We remark that, since $f$ is an isometry, $d F$ commutes with horizontal and vertical lifts, that is,

$$
d F_{s}\left(X_{s}^{H}\right)=\left(d f_{y}(X)\right)_{F(s)}^{H}, \quad d F_{s}\left(X_{s}^{V}\right)=\left(d f_{y}(X)\right)_{F(s)}^{V},
$$

at each point $s=(y, v)$ of $T U$, for every $X \in T_{y} M$. This implies that $F$ is both a local isometry of $(T M, G)$ and a holomorphic mapping with respect to $J$. In particular, $F$ restricts to a local isometry of $T_{r} M$ which is also a CR map. Finally, $F$ is a local symmetry at $t$, since $F(t)=\left(f(x), d f_{x}(u)\right)=t$ and, using (6.16) again, for every $Z=X_{t}^{H}+Y_{t}^{V} \in H_{t}\left(T_{r} M\right)$,

$$
d F_{t}(Z)=\left(d f_{x}(X)\right)_{t}^{H}+\left(d f_{x}(Y)\right)_{t}^{V}=-Z
$$

Finally notice that $f$ can be globally defined when $M$ is complete and simply connected, thus $F$ is also globally defined on $T_{r} M$.

THEOREM 6.2. Let $(M, g)$ be a Riemannian manifold with constant curvature $K$ and dimension $n \geq 3$. Fix $r>0, \lambda>0$, and consider the CR manifold $\left(T_{r} M, H\left(T_{r} M\right), J_{\lambda}\right)$ as above. Then:

(a) $T_{r} M$ is spherical if and only if $\lambda^{2}+K r^{2}=0$;

(b) the metric $g_{\eta_{\lambda}}$ is Sasakian if and only if $\lambda^{2}-K r^{2}=0$;

(c) when $\left(T_{r} M, H\left(T_{r} M\right), J_{\lambda}, g_{\eta_{\lambda}}\right)$ is not Sasakian, its Boeckx invariant is

$$
I=\frac{\lambda^{2}+K r^{2}}{\left|\lambda^{2}-K r^{2}\right|} .
$$

Hence, when $K \neq 0$, each $T_{r} M$ admits a one-parameter family $\left(H\left(T_{r} M\right), J_{\lambda}, \eta_{\lambda}\right)$ of locally (globally for a complete, simply connected $M$ ) CR-symmetric nonhomothetic pseudo-Hermitian structures. If $K<0$, exactly one of the underlying $C R$ structures is spherical. 
PROOF. We begin by computing the spectrum of the operator $h=\frac{1}{2} \mathcal{L}_{\xi} \varphi$ where $\varphi$ is the $f$-structure extending $J:=J_{\lambda}$ on $T\left(T_{r} M\right)$ by $\varphi(\xi)=0$. Fix a point $t=(x, u)$ and consider a holomorphic vector of the form $Z=X_{t}^{V}$ with $g_{x}(X, u)=0$. We shall verify that $X_{t}^{V}$ is an eigenvector of $h_{t}$. Indeed we shall compute $2 h\left(X^{t}\right)=$ $\left[\xi, J X^{t}\right]-J\left[\xi, X^{t}\right]$ and then evaluate at $t$. Here $X^{t}$ is a tangential lift extending $Z$ as in the preceding proof. Now observe that

$$
\left[\xi, J X^{t}\right]_{t}-J\left[\xi, X^{t}\right]_{t}=-\frac{1}{\lambda}\left[\xi, X^{0}\right]_{t}-J\left[\xi, X^{t}\right]_{t}=-\frac{1}{\lambda}\left[\xi, X^{H}\right]_{t}-J\left[\xi, X^{V}\right]_{t} .
$$

In a coordinate neighbourhood $\left(x^{i}, v^{i}\right)$ around $t$,

$$
\left[\xi, X^{V}\right]_{t}=\frac{2}{\lambda}\left\{\left(D_{u} X\right)_{t}^{V}-X_{t}^{H}\right\}
$$

using (6.7), whence

$$
J\left[\xi, X^{V}\right]_{t}=-\frac{2}{\lambda}\left\{\frac{1}{\lambda}\left(D_{u} X\right)_{t}^{H}+\lambda X_{t}^{V}\right\} .
$$

On the other hand, since $(M, g)$ has constant curvature $K,(6.13)$ yields

$$
\frac{1}{\lambda}\left[\xi, X^{H}\right]_{t}=\frac{2}{\lambda^{2}}\left\{\left(D_{u} X\right)_{t}^{H}+K r^{2} X_{t}^{V}\right\} .
$$

Thus, coming back to (6.17), we get

$$
h\left(X_{t}^{V}\right)=\frac{\lambda^{2}-K r^{2}}{\lambda^{2}} X_{t}^{V} .
$$

Since $h$ anticommutes with $J$, from this it also follows that

$$
h\left(X_{t}^{H}\right)=-\frac{\lambda^{2}-K r^{2}}{\lambda^{2}} X_{t}^{H}
$$

for any $X \in T_{x} M$. Thus according to (6.2) we can conclude that the spectrum of $h$ is $\left\{0, \pm\left(\lambda^{2}-K r^{2}\right) / \lambda^{2}\right\}$. Since $T_{r} M$ is a CR manifold, assertion (b) follows directly. To prove (a), we need to compute the Webster scalar curvature of $T_{r} M$. To this end, we compute the scalar curvature of $g_{\eta}$, where $\eta:=\eta_{\lambda}$. We denote by $\nabla^{\prime}$ the LeviCivita connection and by $R^{\prime}$ the curvature tensor of $g_{\eta}$. Recall that $g_{\eta}$ is the restriction of the $g$-natural metric $G$ in (6.15); in particular, we remark that $\pi:\left(T_{r} M, g_{\eta}\right) \rightarrow$ $\left(M,\left(\lambda^{2} / 4 r^{2}\right) g\right)$ is a Riemannian submersion. By standard arguments, we see that the fibres of $\pi$ are totally geodesic and of constant curvature 4 . Indeed, we have the formula

$$
\begin{aligned}
R^{\prime}\left(X_{t}^{V}, Y_{t}^{V}\right) Z_{t}^{V} & =\frac{1}{r^{2}}\left\{g_{x}(Y, Z) X_{t}^{V}-g_{x}(X, Z) Y_{t}^{V}\right\} \\
& =4\left\{g_{\eta}\left(Y_{t}^{V}, Z_{t}^{V}\right) X_{t}^{V}-g_{\eta}\left(X_{t}^{V}, Z_{t}^{V}\right) Y_{t}^{V}\right\}
\end{aligned}
$$


Here $t=(x, u)$ and $X, Y, Z \in T_{x} M$ are orthogonal to $u$. Using the Gauss equation, this formula can be derived from the fact that $\bar{R}\left(X^{V}, Y^{V}\right) Z^{V}=0$, which holds for the curvature of any $g$-natural metric on $T M$ of type $G=a g^{S}+b g^{h}+c g^{v}$ (see [2]). Now the scalar curvature $\tau$ of $\left(T_{r} M, g_{\eta}\right)$ is related to the scalar curvatures $\tau_{M}, \hat{\tau}$ of $\left(M,\left(\lambda^{2} / 4 r^{2}\right) g\right)$ and of the fibres of $\pi$ by

$$
\tau=\tau_{M}+\hat{\tau}-\|A\|^{2}
$$

where $A$ is the O'Neill fundamental horizontal tensor field of the submersion $\pi$ (see, for example, [16]). To compute $\|A\|^{2}$, we fix a point $t=(x, u)$ and an orthonormal frame $\left\{(1 / r p) u, X_{1}, \ldots, X_{n}\right\}$ of $\left(T_{x} M, p^{2} g\right)$ where $p:=\lambda / 2 r$. Then we can consider the orthonormal basis $\left\{(1 / r p) u^{H},\left(X_{1}\right)_{t}^{H}, \ldots,\left(X_{n}\right)_{t}^{H}, \lambda\left(X_{1}\right)_{t}^{V}, \ldots, \lambda\left(X_{n}\right)_{t}^{V}\right\}$ of $\left(T_{t}\left(T_{r} M\right), g_{\eta}\right)$. Now we take into account the formula

$$
\nabla_{X_{t}^{H}}^{\prime} Y^{t}=\frac{1}{2 \lambda^{2}}\left(R_{x}(u, Y) X\right)_{t}^{H}+\left(D_{X} Y\right)_{t}^{t}
$$

which can be derived from Gauss's formula for $T_{r} M$ and the expression for the LeviCivita connection of $(T M, G)$ (see [2]). This formula yields

$$
A_{X_{i}^{H}} X_{j}^{V}=\frac{K}{2 \lambda^{2} p^{2}} \delta_{j}^{i} u^{H}, A_{u^{H}} X_{i}^{V}=-\frac{K r^{2}}{2 \lambda^{2}} X_{i}^{H} .
$$

Thus

$$
\begin{aligned}
\|A\|^{2} & =\lambda^{2} \sum_{i j} g_{\eta}\left(A_{X_{i}^{H}} X_{j}^{V}, A_{X_{i}^{H}} X_{j}^{V}\right)+\frac{\lambda^{2}}{r^{2} p^{2}} \sum_{i} g_{\eta}\left(A_{u^{H}} X_{i}^{V}, A_{u^{H}} X_{i}^{V}\right) \\
& =2 n \frac{K^{2} r^{4}}{\lambda^{4}} .
\end{aligned}
$$

Hence, using (6.20), we obtain

$$
\tau=4 n(n+1) \frac{K r^{2}}{\lambda^{2}}+4 n(n-1)-2 n \frac{K^{2} r^{4}}{\lambda^{4}}
$$

which in turn yields the following formula for the Webster scalar curvature

$$
\rho=4 n^{2}\left(1+\frac{K r^{2}}{\lambda^{2}}\right) \text {. }
$$

Hence, since the metric $g_{\eta}$ is locally CR-symmetric, assertion (a) follows from Theorem 4.4. The determination of the Boeckx invariant is an immediate consequence of (6.18) and (4.9). 
Proof of COROLlary 1.5 . Let $(M, g)$ be a Riemannian manifold with constant curvature $K$ and dimension $n \geq 3$. Assertions (a) and (b) and the uniqueness assertions are direct consequences of (a) and (b) in Theorem 6.2, setting $\lambda=1$. To prove (c), first we remark that when $K=0$ then $\left(T_{r} M, H\left(T_{r} M\right), J, g_{\eta}\right)$ are all non-Sasakian and that the Boeckx invariant actually does not depend on $r$, namely $I=1$. Conversely, assuming that the pseudo-Hermitian manifolds $T_{r} M$ are all locally homothetic, we see from (a) and (b) and the uniqueness assertions that both $K>0$ and $K<0$ must be excluded.

\section{References}

[1] M. T. K. Abbassi, 'Note on the classification theorems of $g$-natural metrics on the tangent bundle of a Riemannian manifold (M, g)', Comment. Math. Univ. Carolin. 45(4) (2004), 591-596.

[2] M. T. K. Abbassi and M. Sarih, 'On Riemannian $g$-natural metrics of the form $a g^{s}+b g^{h}+c g^{v}$ on the tangent bundle of a Riemannian manifold (M, g)', Mediterr. J. Math. 2(1) (2005), 19-43.

[3] D. E. Blair, Riemannian Geometry of Contact and Symplectic Manifolds, Progress in Mathematics, vol. 203 (Birkhäuser, Boston, 2002).

[4] D. E. Blair, T. Koufogiorgos and B. J. Papantoniou, 'Contact metric manifolds satisfying a nullity condition', Israel J. Math. 91 (1995), 189-214.

[5] D. E. Blair and L. Vanhecke, 'Symmetries and $\varphi$-symmetric spaces', Tohoku Math. J. 39 (1987), 373-383.

[6] E. Boeckx, 'A class of locally $\varphi$-symmetric contact metric spaces', Arch. Math. 72 (1999), 466-472.

[7] 'A full classification of contact metric $(k, \mu)$-spaces', Illinois J. Math. 44(1) (2000), 212-219.

[8] _ 'Contact-homogeneous locally $\varphi$-symmetric manifolds', Glasg. Math. J. 48(1) (2006), 93-109.

[9] E. Boeckx and J. T. Cho, ' $\eta$-parallel contact metric spaces', Differential Geom. Appl. 22 (2005), 275-285.

[10] R. L. Bryant, 'Bochner-Kähler metrics', J. Amer. Math. Soc. 14(3) (2001), 623-715 (electronic).

[11] D. Burns and S. Shnider, 'Spherical hypersurfaces in complex manifolds', Invent. Math. 33(3) (1976), 223-246.

[12] S. S. Chern and J. K. Moser, 'Real hypersurfaces in complex manifolds', Acta Math. 133 (1974), 219-271.

[13] J. T. Cho, 'Geometry of contact strongly pseudo-convex CR-manifolds', J. Korean Math. Soc. 43(5) (2006), 1019-1045.

[14] L. David, 'Weyl connections and curvature properties of CR manifolds', Ann. Global Anal. Geom. 26(1) (2004), 59-72.

[15] A. Ghosh and R. Sharma, 'On contact strongly pseudo-convex integrable CR manifolds.', J. Geom. 66(1-2) (1999), 116-122.

[16] M. Falcitelli, S. Ianus and A. M. Pastore, Riemannian Submersions and Related Topics (World Scientific, River Edge, NJ, 2004).

[17] D. Feldmueller and R. Lehmann, 'Homogeneous CR-hypersurface-structures on spheres', Ann. Sc. Norm. Super. Pisa Cl. Sci. 14(4) (1987), 513-525.

[18] J. A. Jiménez and O. Kowalski, 'The classification of $\varphi$-symmetric Sasakian manifolds', Monatsh. Math. 115 (1993), 83-98.

[19] W. Kaup and D. Zaitsev, 'On symmetric Cauchy-Riemann manifolds', Adv. Math. 149 (2000), 145-181.

[20] S. Kobayashi and K. Nomizu, Foundations of Differential Geometry, Vol. I (Interscience Publishers, New York, 1963). 
[21] O. Kowalski and M. Sekizawa, 'On tangent sphere bundles with small or large constant radius', Ann. Global Anal. Geom. 18(3-4) (2000), 207-219.

[22] M. Matsumoto and S. Tanno, 'Kählerian spaces with parallel or vanishing Bochner curvature tensor', Tensor (N.S.) 27 (1973), 291-294.

[23] K. Sakamoto and Y. Takemura, 'On almost contact structures belonging to a CR-structure', Kodai Math. J. 3 (1980), 144-161.

[24] _ _ 'Curvature invariants of CR-manifolds', Kodai Math. J. 4 (1981), 251-265.

[25] T. Takahashi, 'Sasakian $\varphi$-symmetric spaces', Tohoku Math. J. 29 (1977), 91-113.

[26] N. Tanaka, 'On non-degenerate real hypersurfaces, graded Lie algebras and Cartan connections', Japan. J. Math. 20 (1976), 131-190.

[27] S. Tanno, 'Variational problems on contact Riemannian manifolds', Trans. Amer. Math. Soc. 314 (1989), 349-379.

[28] $\quad$ 'The standard CR structure on the unit tangent bundle', Tohoku Math. J. 44(4) (1992), 535-543.

[29] — 'Pseudo-conformal invariants of type $(1,3)$ of CR manifolds', Hokkaido Math. J. 20(2) (1991), 195-204.

[30] Y. Tashiro, 'On contact structures of tangent sphere bundles', Tohoku Math. J. 21 (1969), 117-143.

[31] S. M. Webster, 'Pseudo-Hermitian structures on a real hypersurface', J. Differential Geom. 13(1) (1978), 25-41.

[32] _ _ 'On the pseudo-conformal geometry of a Kähler manifold', Math. Z. 157 (1977), 265-270.

G. DILEO, Dipartimento di Matematica, Università di Bari, Via E. Orabona 4, 70125 Bari, Italy

e-mail: dileo@dm.uniba.it

A. LOTTA, Dipartimento di Matematica, Università di Bari, Via E. Orabona 4, 70125 Bari, Italy

e-mail: lotta@dm.uniba.it 